\title{
Assessing the Impact of Aerodynamic Modelling on Manoeuvring Aircraft
}

\author{
A. Da Ronch * \\ University of Southampton, Southampton, SO17 1BJ, United Kingdom \\ A. J. McCracken † N. D. Tantaroudas $\ddagger$ and K. J. Badcock $\S$ \\ University of Liverpool, Liverpool, L69 3GH, United Kingdom \\ H. Hesse " and R. Palacios $\|$ \\ Imperial College, London, SW7 2AZ, United Kingdom
}

\begin{abstract}
This paper investigates the impact of aerodynamic models on the dynamic response of a free-flying aircraft wing. Several options for the aerodynamics are evaluated, from two-dimensional thin aerofoil aerodynamics and unsteady vortex-lattice method up to computational fluid dynamics. A nonlinear formulation of the rigid body dynamics is used in all cases. Results are generated using a numerical framework that will allow in the near future multi-disciplinary fluid/structure/flight analysis. In this paper, flexibility effects are neglected. A validation for fluid/flight models is presented. The well-established approach based on stability derivatives is also used, and is found in good agreement with solutions obtained from linear aerodynamic models. The uncertainties in predicted trajectories of the free-flying wing are, in general, large and attributed to the aerodynamics only. This suggests that a careful control law synthesis should be done to account for uncertainties from modelling techniques.
\end{abstract}

\section{Nomenclature}

$\begin{array}{ll}c & =\text { mean aerodynamic chord } \\ C_{j} & =\text { longitudinal force and moment coefficients } \\ C_{j 0} & =\text { nonlinear static longitudinal force and moment coefficients } \\ \bar{C}_{j_{q}} & =\text { longitudinal force and moment damping coefficients } \\ L, D, M & =\text { lift, drag and pitch moment } \\ I_{z z} & =\text { second moment of inertia } \\ h & =\text { altitude } \\ k & =\text { reduced oscillation frequency } \\ M & =\text { Mach number } \\ R e & =\text { Reynolds number } \\ U_{\infty} & =\text { freestream velocity } \\ \dot{y} & =\text { velocity in vertical direction }\end{array}$

Greek

$\alpha \quad=$ angle of attack

\footnotetext{
${ }^{*}$ Lecturer, Faculty of Engineering and the Environment; A.Da-Ronch@soton.ac.uk. Member AIAA (Corresponding Author).

${ }^{\dagger} \mathrm{PhD}$. Candidate, School of Engineering.

${ }^{\ddagger}$ PhD. Student, School of Engineering.

$\S$ Professor, School of Engineering. Senior Member AIAA.

『Research Associate, Department of Aeronautics. Member AIAA.

$\|$ Senior Lecturer, Department of Aeronautics. Member AIAA.
} 


$\begin{array}{ll}\alpha_{A} & =\text { amplitude of oscillatory motion } \\ \alpha_{0} & =\text { mean angle of attack } \\ \theta_{z} & =\text { pitch angle }\end{array}$

Subscripts

$\begin{array}{ll}L & =\text { lift } \\ D & =\text { drag } \\ m & =\text { pitching moment }\end{array}$

\section{Introduction}

For flight dynamics analysis, a model of the aerodynamic forces and moments dependency on flight and control states is needed. This is often expressed in tabular form. There are several possible sources of data for this aerodynamic database. Flight testing is the most accurate but also the most expensive of these methods. ${ }^{1}$ Wind-tunnel testing of scaled models is cheaper than flight testing. However, blockage, scaling and Reynolds number effects together with support interference limits the proper modelling of the full scale aircraft behaviour. ${ }^{2}$ The third approach combines data sheets, linear aerodynamic theory and empirical relations. ${ }^{3}$ Due to simplicity, this method is in widespread use and is a common choice to obtain aerodynamic characteristics in the conceptual design stage. In the absence of a background database, empiricism is limited when confronted with novel configurations and flight conditions dominated by nonlinear aerodynamic effects.

A possible compromise between the high-fidelity/high-cost of testing and low-fidelity/low-cost of semiempirical approaches is Computational Fluid Dynamics (CFD), which represents the state of the art in predicting nonlinear flow physics. However, the generality realised in a CFD simulation comes at the expense of computational cost. To generate the aerodynamic database of forces and moments for the expected flight envelope, surrogate models are often used to reduce the number of calculations. ${ }^{4}$

The concept of stability or aerodynamic derivatives was introduced by $\operatorname{Bryan}^{5}$ in 1911 and remains essentially unchanged as the conventional model for the representation of the aerodynamic loads in the equations of motion. It is assumed that the aerodynamic forces and moments are a function of the instantaneous values of the disturbance velocities, control angles and their rates. The dependence of the forces and moments on these variables is obtained by a Taylor series expansion, discarding higher order terms. For slow motions at low angle of attack, the static derivatives are generally sufficient to model the aerodynamic loads. At higher angles of attack and rates, the inclusion of dynamic derivatives in the aerodynamic model can have a significant effect on the calculated stability characteristics of an airframe. The prediction of dynamic derivatives requires the ability to compute the aerodynamic response to time-dependent prescribed motions which are used to excite the aerodynamics of interest. In Ref., ${ }^{6}$ a comparison of dynamic derivatives computed using CFD and measured in wind-tunnel experiments was documented. For the two aircraft configurations studied, it was observed that the dynamic derivatives vary with motion and flow parameters, and these variations cannot be reconciled with the stability derivatives model.

A common technique for the prediction of dynamic derivatives relies on harmonic forced-oscillation tests. After the decay of initial transients, the nature of the aerodynamic loads becomes periodic. A time-domain simulation of this problem requires significant computational effort. Several oscillatory cycles have to be simulated to obtain a harmonic aerodynamic response, and a time-accurate solution requires a small timestep increment to accurately capture the flow dynamics. ${ }^{6}$ Time-domain calculations support a continuum of frequencies up to the frequency limits given by the temporal and spatial resolution, but dynamic derivatives are computed at the frequency of the applied sinusoidal motion. Reference ${ }^{7}$ considered a frequency-domain formulation to obtain a good estimate of the derivatives at reduced computational cost. The harmonic balance and the linear frequency domain methods were compared in terms of accuracy and computational time. Compared to time-domain simulations, an order of magnitude reduction in computational cost was achieved, and satisfactory predictions were obtained for cases with a narrow frequency spectrum and moderate amplitudes.

A review of the applications and limitations of tabular models for flight dynamics analysis was reported in Ref. ${ }^{8}$ Tables were derived from a hierarchy of aerodynamic models, which included numerical tools, windtunnel measurements, and flight tests data. Five test cases were presented, featuring some unconventional 
aircraft concepts. Confronted with the limitations and shortcomings of the conventional stability derivative model, researchers have looked at alternative models. For example, in Ref. ${ }^{9}$ four alternative models were tested for the representation of the unsteady and nonlinear aerodynamic loads around the X-31 aircraft in transonic flow. Issues with model robustness and cost of model generation were discussed. A nonlinear model based on indicial functions and a time-dependent surrogate model were indicated as the best options.

The objectives of this work are two-fold. First, a solver for the coupled aerodynamic-flight dynamics simulation of a vehicle with moving control surfaces will be developed and validated. Aerodynamic options range from linear unsteady two-dimensional theory up to CFD, featuring Euler and unsteady RANS models. The impact of aerodynamic modelling on a set of manoeuvres for forced motions and free flight conditions is investigated. Based on this validated framework, aerodynamic tabular models will be assessed in terms of accuracy for critical loads conditions, e.g., off-design conditions or at the edges of the flight envelope. The second objective is the extension of the approach to model reduction, ${ }^{10}$ now applied to aeroelastic cases, to flight dynamics degrees of freedom. This will enable the reduction of a large nonlinear system to a smaller linear/nonlinear system, with most of the important system dynamics preserved, which is adequate to speed up the simulation process in the design validation stage. Through the design of a control system, uncertainties in aerodynamic predictions are evaluated in terms of control surfaces deflection required to either track a predefined trajectory or to counteract external disturbances, e.g., atmospheric gust. Flexibility effects will be included in future works.

\section{Formulation}

\section{II.A. Aerodynamic Models}

Various aerodynamic models are used in this work, and these are summarised below.

\section{II.A.1. Two-dimensional Thin Aerofoil Theory}

A computationally cheaper alternative to the CFD model is based on strip theory and the incompressible two-dimensional classical theory of Theodorsen. The total aerodynamic loads consist of contributions arising from the section motion, flap deflection and the penetration into a gusty field. The aerodynamic loads due to an arbitrary input time-history are obtained through convolution against a kernel function. For the influence of the aerofoil motion on the loads, the Wagner function is used. For the response to an arbitrary gust, the integration uses the Küssner function. Since the assumption is of linear aerodynamics, the effects of the two influences on the aerodynamic forces and moments are added together to find the variation of the forces and moments in time for a given motion and gust. For a practical evaluation of the integral, an exponential approximation is used for the Wagner and Küssner functions. The aerodynamic state vector for this model, $\boldsymbol{w}_{f}$, has dimension 8 , and the formulation in terms of a residual function follows that given in Ref. ${ }^{10}$

\section{II.A.2. Unsteady Vortex-Lattice Method}

The unsteady vortex lattice method (UVLM) is used in this work to obtain a medium-fidelity threedimensional solution of the unsteady aerodynamics. ${ }^{11,12}$ The UVLM is a geometrically nonlinear method in which the shape of a force-free wake is obtained as part of the solution procedure. It therefore accurately captures the aerodynamic lags over a large range of reduced frequencies at low flight velocities which makes this method suitable for the analysis of very flexible aircraft. ${ }^{13}$

In the UVLM, vortex-ring quadrilateral elements are used to discretize the lifting surfaces and wakes. As described in Ref., ${ }^{14}$ the vorticity distribution of all vortex elements is determined by applying the nonpenetration boundary condition over the bound vortex panels along the lifting surfaces. The Biot-Savart law is used to compute the induced velocities over the normal vector of each vortex ring. Inputs to the aerodynamic problem, such as deployment of control surfaces, gust-induced velocities, wing deformations and rigid-body motions, are easily introduced through non-vortical velocities applied normal to each surface panel. Once the vorticity distribution is determined at each time step, the aerodynamic pressures can be computed using Bernoulli's equation. The resulting aerodynamic loads are finally converted into forces and moments at the beam nodes assuming coincident meshes and rigid cross-sections. ${ }^{11}$ 


\section{II.A.3. Computational Fluid Dynamics}

The CFD code used is the Parallel Meshless solver of the University of Liverpool which solves the Euler, laminar and Reynolds-Averaged Navier-Stokes equations (with the Spalart-Almaras turbulence model) on a point cloud. The solver is summarised in Ref. ${ }^{15}$ The unknowns are stored at each (star) point, and a cloud of surrounding points is defined for the spatial discretisation. The definition of this stencil is a non-trivial problem which is solved using the method described in Ref., ${ }^{16}$ which exploits information from point connectivity in underlying component meshes to guide the search ellipses for the stencil. Successive application of this method allows the stencils to be updated for moving geometries, providing great flexibility for the problems that can be computed.

Osher's approximate Riemann solver is used to evaluate the convective fluxes at the mid point between the star point and every other point in the stencil. A least squares reconstruction is calculated at each star point to estimate the gradients of the flow unknowns there also. These gradients are then used to provide a high order reconstruction of the interface values for the Riemann problem, with the reconstruction limited to avoid oscillations by the Barth and Jespersen limiter. A least squares reconstruction of the mid point fluxes is then used to calculate the gradients required for the residual. The star point gradients are used to calculate the terms needed for the viscous fluxes, which are averaged to the mid-points and then reconstructed as for the convective fluxes to obtain the required viscous contributions to the residual. At each stage it is straightforward to use the chain rule to calculate the Jacobian matrix contributions analytically. The steady state CFD solver uses an implicit method which drives convergence using the Jacobian matrix, solving a linear system for the updates with a preconditioned Krylov sparse matrix solver. For moving body problems a number of options for moving the point clouds are available. Time accurate calculations are done using the pseudo time stepping method, which solves a modified steady state problem for the updated solution at each new time step. The treatment of gust encounters is described in Ref. ${ }^{17}$

To model the trailing-edge control surface, two component meshes have been generated. The two meshes are then converted to point clouds as shown in Fig. 1(a). The preprocessor removes any points which are determined to be inside any boundaries following the overlap and the stencils are chosen based on the method above. The resulting point cloud is shown in Fig. 1(b).

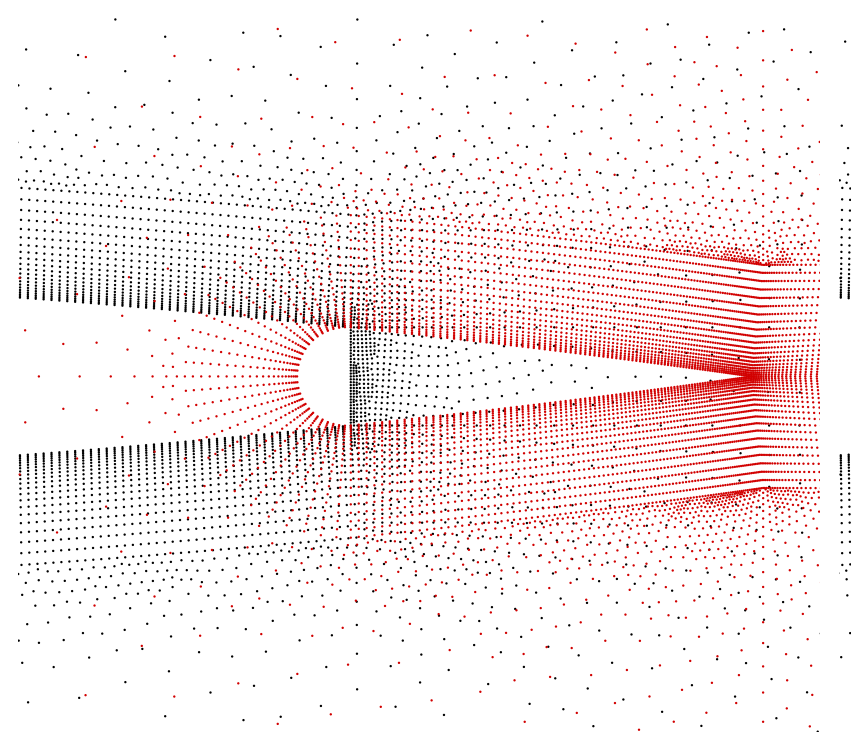

(a) Before

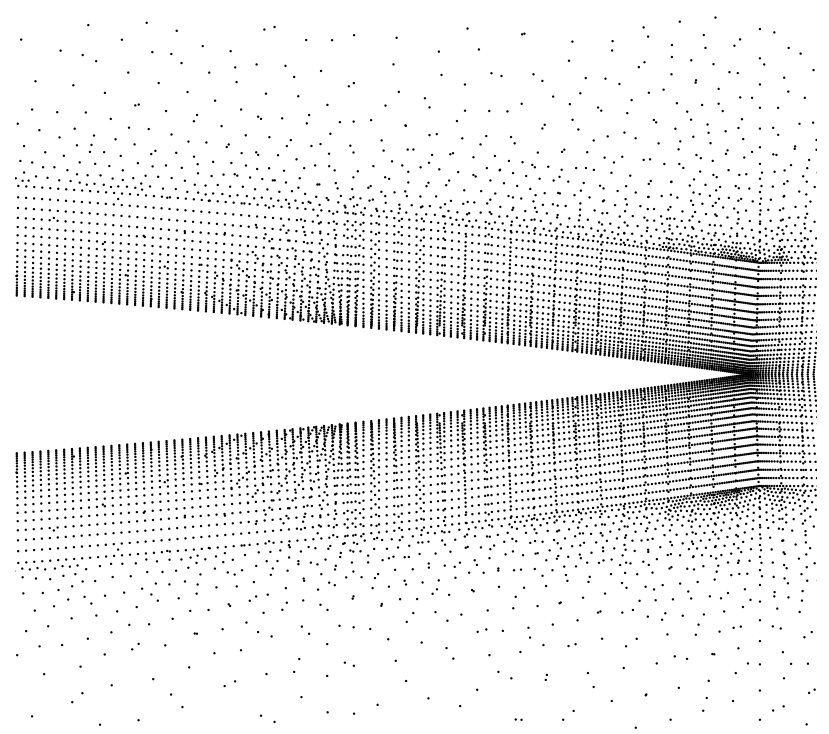

(b) After

Figure 1. Body of a NACA 0012 aerofoil section (black) and trailing-edge flap (red) point clouds before and after running the preprocessor

\section{II.A.4. Stability Derivatives Approach}

For the application of the aerodynamic stability derivative model, the load and moment coefficients are considered as consisting of a steady and an unsteady component expanded as shown in Eq. (1) for a pitching 
motion

$$
C_{j}(t)=C_{j_{0}}(t)+\bar{C}_{j_{q}} q(t) \frac{c}{U_{\infty}}
$$

The subscript $j$ represents the force or moment of interest (e.g. L, D, M), the zero subscript term is the steady value at time $t$ and the term $q(t)$ is the rate of change of incidence at time $t$. The dynamic stability derivative term $\bar{C}_{j_{q}}$ in this example represents how the force or moment coefficient changes with the pitch rate. The first Fourier coefficients of the time history of the response correspond to the values of the stability derivatives.

The dynamic derivatives are calculated in this work using two different methods. The first approach is to simulate a forced periodic motion as described in Ref. ${ }^{6}$ This simulation is carried out using the UVLM and CFD solvers. The derivatives are then calculated from the response to forced motions. The second approach is to identify the derivatives from the free-response simulations. The stability derivatives, in this case, are calculated by a least-squares fit to the available time-domain data.

\section{II.B. Coupled Structure/Flight Models}

The dynamics of flexible aircraft subject to large manoeuvres are modelled using a geometrically-exact composite beam formulation which has been extended to account for the vehicle rigid-body dynamics. This approach is described in detail in Ref. ${ }^{18}$ and takes advantage of the slenderness of aircraft sub-components, increasingly made of lightweight composite materials. The possibly large elastic deformations are described in terms of one-dimensional beam elements on a moving (body-attached) frame of reference. The resulting elastic degrees of freedom, $\boldsymbol{\eta}$, are the displacements and rotations at the element nodes, whereby the Cartesian rotation vector was used in this work to parametrise the finite rotations due to large deformations. ${ }^{19}$ The overall dynamics of the aircraft are described in terms of the translational and angular velocities, $\boldsymbol{\nu}$, of the body-attached reference frame.

The dynamic equations of the coupled structural/flight dynamic model are then written as

$$
\mathcal{M}(\boldsymbol{\eta})\left\{\begin{array}{c}
\ddot{\boldsymbol{\eta}} \\
\dot{\boldsymbol{\nu}}
\end{array}\right\}+\left\{\begin{array}{c}
\boldsymbol{Q}_{\text {gyr }}^{S} \\
\boldsymbol{Q}_{\text {gyr }}^{F}
\end{array}\right\}+\left\{\begin{array}{c}
\boldsymbol{Q}_{\text {stif }}^{S} \\
\mathbf{0}
\end{array}\right\}=\left\{\begin{array}{c}
\boldsymbol{Q}_{\text {ext }}^{S} \\
\boldsymbol{Q}_{\text {ext }}^{F}
\end{array}\right\}
$$

where the discrete mass matrix $\mathcal{M}$ and the discrete gyroscopic, elastic and external generalised forces, $\boldsymbol{Q}_{g y r}$, $\boldsymbol{Q}_{\text {stif }}$ and $\boldsymbol{Q}_{\text {ext }}$, respectively, are obtained through a finite element discretisation of the primary variables as presented in Ref. ${ }^{18}$ The structural and flight dynamic components (denoted by superscripts $S$ and $F$ ) have also been identified in the generalised discrete forces.

The resulting differential equation, Eq. (2), couples the nonlinear beam dynamics and the nonlinear rigid-body dynamics through the fully-populated mass matrix, $\mathcal{M}(\boldsymbol{\eta})$, and the discrete gyroscopic forces, $\boldsymbol{Q}_{g y r}(\boldsymbol{\eta}, \dot{\boldsymbol{\eta}}, \boldsymbol{\nu})$. Additional coupling occurs through the external forces $\boldsymbol{Q}_{\text {ext }}(\boldsymbol{\eta}, \dot{\boldsymbol{\eta}}, \boldsymbol{\nu})$ which can include gravity, thrust and the aerodynamic loads from the different models presented above. Equation (2) is finally time-marched using an implicit, constant-acceleration Newmark integration scheme. ${ }^{20}$ In this work the multi-disciplinary framework is presented for rigid configurations which simplifies Eq. (2) to a standard flight dynamics model. Future work will demonstrate the approach for the fully coupled fluid/structure/flight problem.

\section{Multi-disciplinary Framework}

A current key issue in aerospace engineering is the understanding of the complex interactions between multiple disciplines. Here, we are interested in developing a framework for high-fidelity numerical simulations for coupled fluid/structure/flight models. With the previous sections on the underlying models for each discipline as background, the development of a multi-disciplinary framework is described next.

\section{III.A. Code Modules}

The successful development of a multi-disciplinary framework depends not only on the algorithms for each component of the vehicle design and analysis, but also on the ability to provide an interface between existing codes for straightforward coupling and scripting. This facilitates the implementation of coupled procedures, as explained below. 
The flexibility to provide an object-oriented interface to different programming languages makes Python ${ }^{\mathrm{a}}$ a clear choice for creating a modular and easy to use environment. The approaches used to create an automatic interface for Fortran 90 and $\mathrm{C}$ languages are explained in this section. This is exemplified, in particular, for the CFD code which is written entirely in C and uses Message Passing Interface ${ }^{\mathrm{b}}$ (MPI) for parallel computing, and for the structure/fluid code written in Fortran 90 language.

\section{III.A.1. Wrapping C Language with Python}

The C-based CFD code was interfaced to Python using f2py. ${ }^{21}$ This tool can be used as an automatic interface generator for both Fortran and $\mathrm{C}$ languages. The first step consists of creating an interface file, for example named wrap_cfd.c, written in $\mathrm{C}$ language allowing easy access to any $\mathrm{C}$ function from Python and avoiding the need to modify directly the source code. This file is unique to the lower level language and is used to transfer information between the flow solver and Python, e.g. returning surface pressure. As a second step, the prototypes of the $\mathrm{C}$ functions included in the interface file that will be directly exposed to Python are written to a signature file with extension pyf (wrap_cfd.pyf). The syntax of this file is a mixture of Fortran 77 statements and Python, but it is extremely easy to set up manually. As a final step, given the signature file, f2py creates the $\mathrm{C}$ code wrappers and compiles a shared dynamic library (.so file) that can be imported directly into Python as a normal module.

The ability to execute a computationally intensive code across multiple processors is essential to tackle realistic aircraft applications. This was done using mpi4py ${ }^{22}$ that provides an object-oriented approach to MPI for the Python programming language. Whereas this enables Python to run on distributed parallel machines, most of the MPI communication calls are still executed within the CFD code that was using them in the first place. Compared to the CFD code alone, the overhead for using mpi4py was found negligible, as shown in Table 1 for a Goland wing consisting of 187,523 points. Deviations from a linear speed-up are attributed to increased communication overhead for a problem of such small size.

Table 1. Parallel speed-up comparison using the CFD code alone and the mpi4py package

\begin{tabular}{lcc}
\hline \hline Processors & CFD alone & CFD \& mpi4py \\
\hline 1 & 1.00 & 1.00 \\
2 & 1.69 & 1.68 \\
4 & 2.17 & 2.12 \\
8 & 2.30 & 2.24 \\
\hline \hline
\end{tabular}

\section{III.A.2. Wrapping Fortran 90 Language with Python}

The structure/flight code considered in this study is written in Fortran 90 language. As mentioned in the previous section, f2py can be used to automatically generate an interface to wrap Fortran/C with Python. An alternative approach was used, which avoids creating intermediate files while retaining the same flexibility as above. Given a set of subroutines from the original source code that is essential to add new functionalities to the framework, an interface file written in Fortran 90 is prepared. This file avoids modifying directly the source code and allows exchanging information between the low level code and Python, e.g. structural deformations or rigid body motion. The next step is to compile the source files into a shared dynamic library (. so file) using any available Fortran compiler. The shared library can be imported using the ctypes ${ }^{\mathrm{c}}$ library for Python, which exports the cdll to load dynamic link libraries.

\section{III.B. Fluid-Structure Interface}

The generation of Python modules for each disciplines is straightforward, and allows accessing each function we have chosen to wrap as well as passing information to/from the low level code. Up to this point, however, the discussion has been limited to details for each separate field of the analysis (e.g. structure, flight,

\footnotetext{
${ }^{a}$ http://www.python.org/ [retrieved December 17, 2013]

b ${ }_{\text {http://www.open-mpi.org/ [retrieved December 17, 2013] }}$

chttp://docs.python.org/3.2/library/ctypes.html [retrieved December 17, 2013]
} 
aerodynamics). The interfaces are created in a way that each discipline can either be used as standalone for single discipline analysis or be imported as a module by a higher level class or script that performs a multi-disciplinary analysis.

Since we are interested in creating the capabilities to perform a coupled fluid/structure/flight analysis, a mesh deformation tool is needed to transfer information between the fluid and structural solver. The generality of using any arbitrary fluid and structural solvers introduces the difficulty of dealing with noncoincident and non-overlapping discretisations. As one example, beam elements for the structure and a three-dimensional CFD grid are common.

The transfer of information between non-matching fluid and structural grids has been an active subject of reseach. The method used here is based on the moving least squares method introduced in Ref. ${ }^{23}$ This method is: a) efficient in terms of memory storage and parallelizable; b) independent of the fluid and structural formulations; c) conservative, e.g. without introducing spurious energy; and d) able to deal with arbitrarily large deformations around complex geometries and interface non-coincident and non-overlapping fluid/structure domains. The linearity of the fluid/structure interface scheme is exploited to transfer aerodynamic loads from the aerodynamic to the structural model, and map structural deformations from the structural to the aerodynamic model at no additional cost. A valuable property of the method is that extrapolation beyond the support of the structural model, which becomes particularly significant if a wing box is being used, is linear.

Results for the surface mesh deformation are shown in Fig. 2 for two geometries. The first geometry is the AGARD 445.6 wing. The structural model from the experimental work of Ref. ${ }^{24}$ consists of 121 points. This was used to map the deformations of the fourth modeshape to the aerodynamic surface that contains 4737 points. The DSTL UAV from Ref. ${ }^{25}$ is the second geometry presented here. A detailed finite element model of the structure is available with 1336 grid points. The main wing and the canted tail are elastically suspended to a rigid fuselage. The aerodynamic model consists of 8310 surface points.

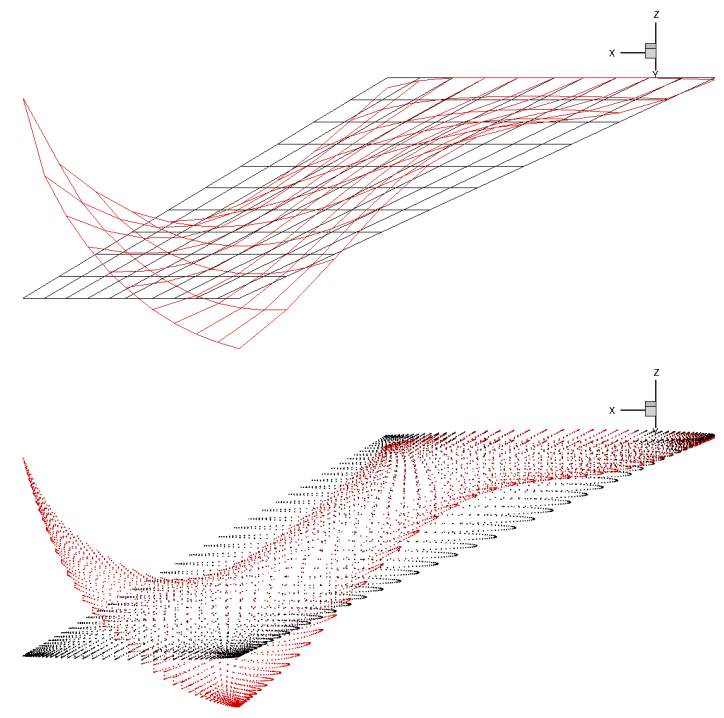

(a) AGARD 445.6 wing

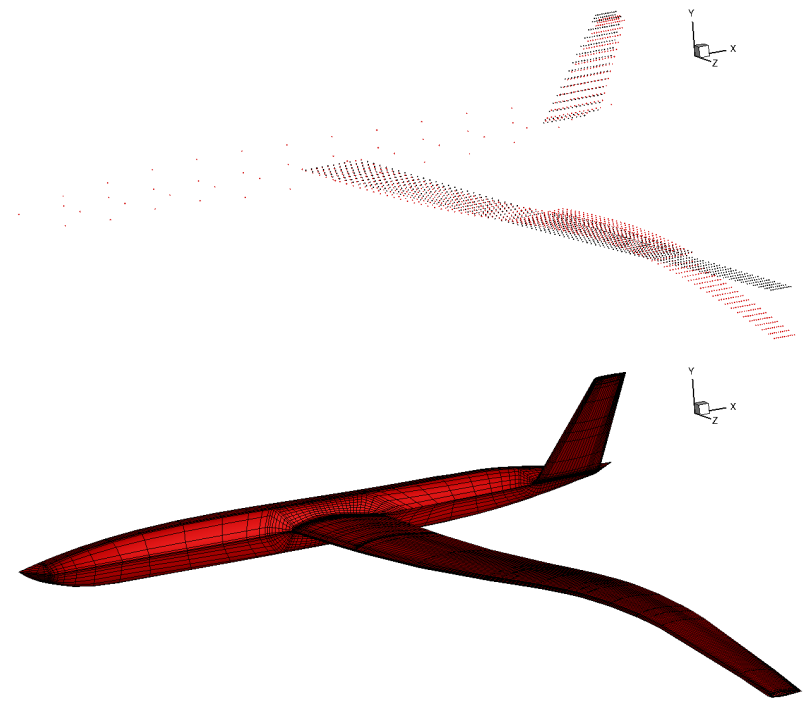

(b) DSTL UAV

Figure 2. Normal structural modeshapes mapped to the aerodynamic mesh; a) fourth modeshape of the AGARD 445.6 wing $(f=91.54 \mathbf{H z})$; and $\mathbf{b})$ second modeshape of the DSTL UAV $(f=2.53 \mathbf{H z})$

The problem of constructing a deformed volume mesh that conforms to the actual deformed surface mesh at every time step in a calculation is here avoided. An efficient and robust method to update the volume mesh based on the inverse distance weighting (IDW) approach ${ }^{26}$ has been implemented in the numerical framework. Illustrative results of the static aeroelastic response of the Goland wing at $M=0.5, \alpha_{\infty}=0.8$ deg, $h=9,000 \mathrm{ft}$ are shown in Fig. 3. In the absence of nonlinear flow features, the good agreement between the solution based on UVLM and CFD is expected. 


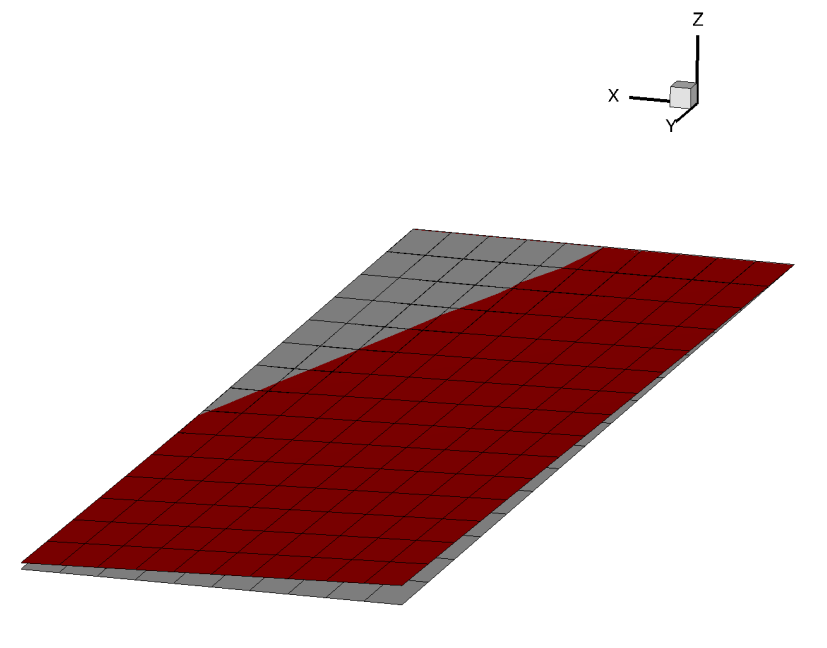

(a) UVLM solution

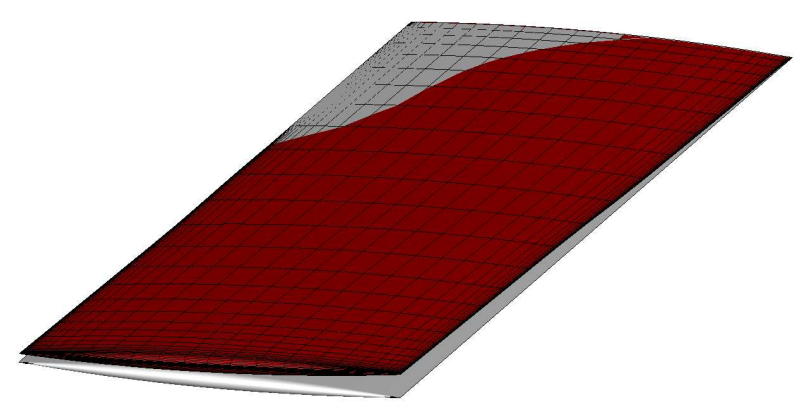

(b) CFD solution

Figure 3. Static aeroelastic solution for the Goland wing test case using the UVLM and the Euler equations $(M=0.5$, $\left.\alpha_{\infty}=0.8 \mathrm{deg}, h=9,000 \mathbf{f t}\right)$

\section{III.C. Coupled Procedures}

A common approach for coupled simulations is based on a staggered procedure, in which separate analysis codes, e.g. fluid and structure, are run in a strictly sequential manner. At each time step, the balance between the energy developed by the fluid forces on the structure and the energy exchanged to the fluid through the interface is not guaranteed. These methods can become numerically unstable by introducing spurious energy.

In the numerical framework developed, a fully implicit partitioned approach is used. The solution sequencing between the fluid and structural models is achieved within the pseudo-time stepping iterations. The models are sequenced in pseudo-time towards perfect sequencing in real time. The same approach is used for the fluid/flight models considered in this paper.

\section{Two-dimensional Wing Section}

To study the impact of aerodynamic models on the characteristics of flying wings, a baseline flying aircraft wing is introduced. The geometry and cross-sectional properties are not based directly on previous test cases available in the literature, but rather selected to setup a flight mechanics model prototype.

The geometry of the flying wing has a constant chord of $1.0 \mathrm{~m}$ and the NACA0012 aerofoil is used for the wing section. Because some of the aerodynamic options used herein are inadequate to realistically capture three-dimensional aerodynamic effects around a finite span wing (e.g. wing tip vortices), the wing is assumed two-dimensional. For the two-dimensional thin aerofoil theory, a unit wing span was used. For the (three-dimensional) UVLM, a preliminary study was performed to numerically evaluate the influence of wing span on the response. The aspect ratio was 1, 10, and 100. Results were found to be independent of the aspect ratio for the two larger values, and an aspect ratio of 10 was then used for all results herein presented. The CFD results in this section are for a two-dimensional solution around the NACA0012 aerofoil, and this allows to use a unit wing span and drastically reduce computational costs.

Wing cross-sectional properties can be found in Table 2. With a flying speed of $50.0 \mathrm{~m} / \mathrm{s}$ at sea level, the free response of the flying wing is studied for an initial angle between flow direction and aerofoil chord of $1 \mathrm{deg}$. First, the flying wing is constrained to freely rotate in pitch only, see Sec. IV.B. Then in Sec. IV.C, the flying wing is let to freely move in the vertical direction and to rotate in pitch. 
Table 2. Reference values of the two-dimensional wing section

\begin{tabular}{lc}
\hline \hline Parameter & Value \\
\hline Elastic axis & $5 \%$ chord \\
Centre of gravity & $5 \%$ chord \\
$\quad$ Inertia properties & \\
Mass per unit length & $10.0 \mathrm{~kg} / \mathrm{m}$ \\
Mass moment of inertia (torsional) & $10.0 \mathrm{~kg} \cdot \mathrm{m}$ \\
Geometry & \\
Chord & $1.0 \mathrm{~m}$ \\
Span & $\infty$ \\
\hline \hline
\end{tabular}

\section{IV.A. Validation}

Starting with a validation of the CFD solver, a comparison is given in Fig. 4 for numerical predictions of dynamic lift and pitching moment coefficients. The test case is for $M=0.3, R e=4 \times 10^{6}, \alpha_{0}=10 \mathrm{deg}$, $\alpha_{A}=10 \mathrm{deg}$, and $k=0.1$, which corresponds to case 8 in Ref. ${ }^{27}$ A preliminary study on grid convergence was performed. The agreement to experimental data for the solution on a cloud of 25,509 points, show in Fig. 5, is satisfactory. Differences could be attributed to the turbulence model used in this study, based on the Spalart-Almaras.

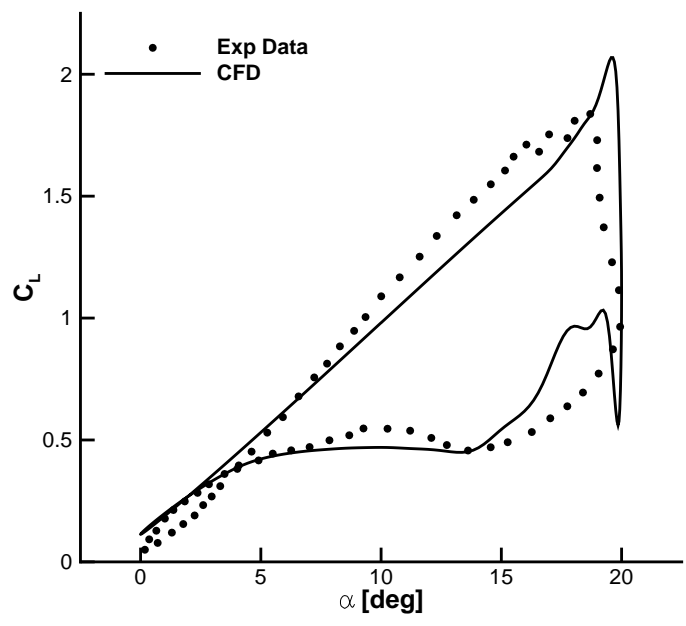

(a) Lift coefficient

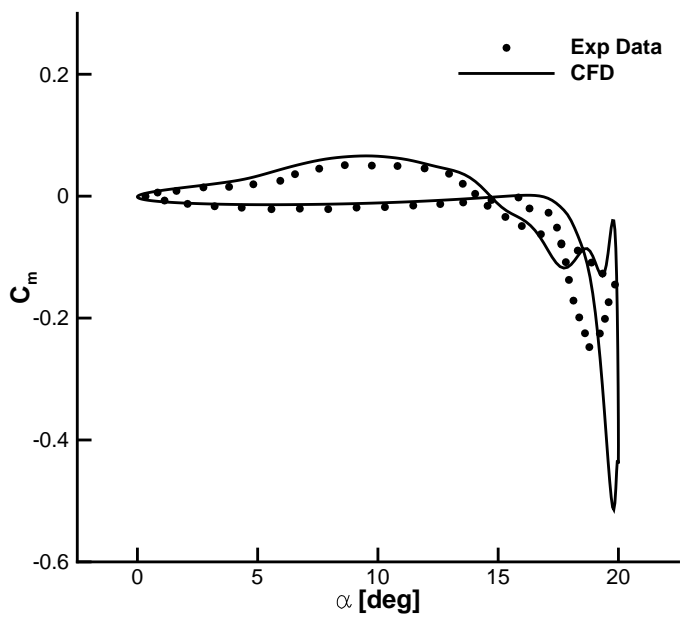

(b) Pitching moment coefficient

Figure 4. Lift and pitching moment coefficients for NACA 0012 aerofoil $\left(M=0.3, R e=4 \times 10^{6}, \alpha_{0}=10\right.$ deg, $\alpha_{A}=10$ deg, and $k=0.1$ ); experimental data are for case 8 in Ref. ${ }^{27}$

\section{IV.B. Free-to-pitch Test Case}

The test case studied here has both horizontal and vertical displacements constrained. The flying wing is only free to rotate about the elastic axis, which is placed at $5 \%$ of the wing chord from the leading edge. The location of the elastic axis relative to the centre of pressure determines the dynamic behaviour of the free-to-pitch wing. With a flying speed of $50 \mathrm{~m} / \mathrm{s}$, thin aerofoil theory suggests that the centre of pressure is at one quarter of the chord behind the leading edge. This guarantees that the configuration is dynamically stable and will be verified by simulations.

The free response of the free-to-pitch wing is investigated using the three aerodynamic models already presented. The time-domain results are shown in Fig. 6. The simulations are driven by initially setting 


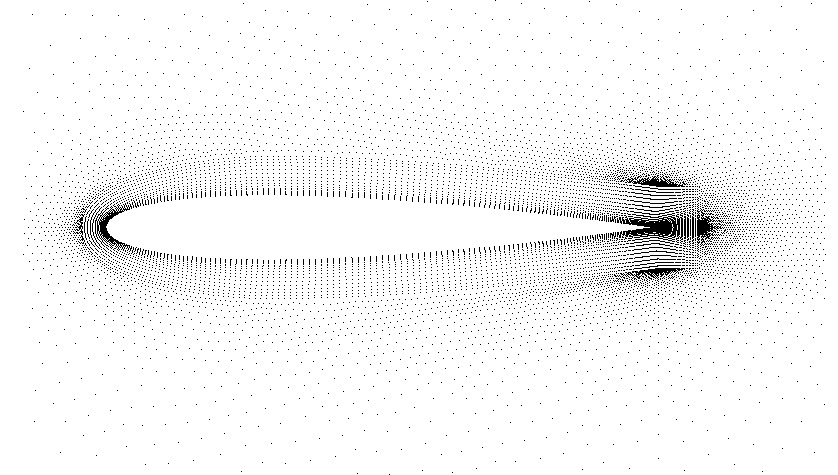

Figure 5. Point distribution for NACA 0012 aerofoil, RANS

the angle between the flow direction and the wing chord to $1 \mathrm{deg}$, positive nose up. With the flow in the horizontal plane, the wing was rotated around the elastic axis by $1 \mathrm{deg}$ in the results computed with thin aerofoil theory and UVLM. As usually done in most CFD codes, the wing section is not rotated and the flow direction is set to $1 \mathrm{deg}$ angle of attack. In general, the choice of the time step in any time-domain simulation is important and, in particular, is critical in a CFD analysis. A study was performed to verify that the CFD solution was independent of the time step used. Two time steps were used (in physical time: $4.9 \cdot 10^{-3} \mathrm{~s}$ and $1.0 \cdot 10^{-3} \mathrm{~s}$; in non-dimensional time: $2.4 \cdot 10^{-1}$ and $5.0 \cdot 10^{-2}$ based on the wing chord and freestream speed). Because no significant differences were found, results are for the larger time step.

Figure 6 shows the time-domain response of the free-to-pitch wing section. Three coupled fluid/flight models are compared. Qualitatively, results confirm the dynamically stable behaviour of the wing, in agreement with low speed aerodynamics theory. The quantitative differences are attributed mainly to differences in the aerodynamic models, since the flight mechanics solver is the same. With no structural damping and stiffness, the aerodynamic model is responsible for the observed oscillatory delay. The actions generated from the fluid around the rotating wing section can be seen as "equivalent" structural damping and stiffness terms.

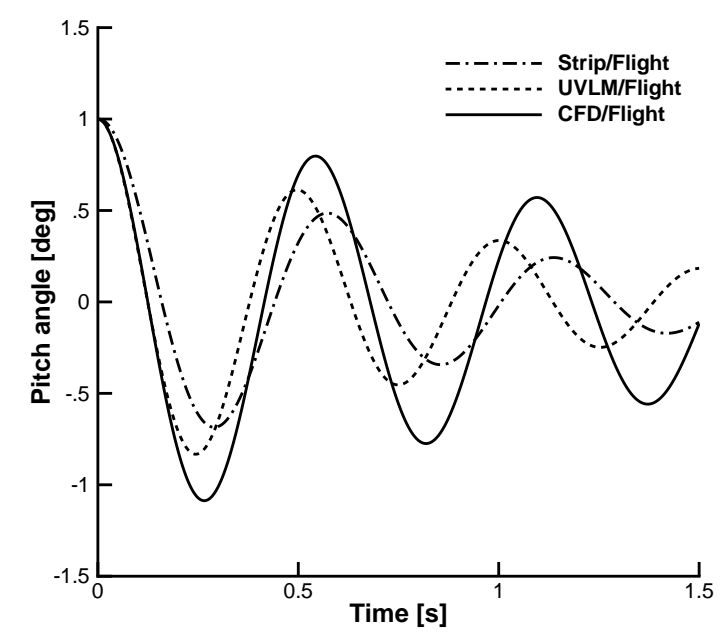

Figure 6. Time-domain response of a free-to-pitch two-dimensional wing section; "Strip" denotes two-dimensional thin aerofoil theory $\left(\alpha_{\infty}=1.0 \mathrm{deg}, U_{\infty}=50.0 \mathrm{~m} / \mathrm{s}, h=0.0 \mathbf{m}\right.$, and $\left.\mathbf{R e}=3.5 \cdot 10^{6}\right)$ 


\section{IV.B.1. Model based on Aerodynamic Stability Derivatives}

A simplified dynamic model of the free-to-pitch wing section studied here is that of a one degree of freedom system. Denote $\theta_{z}$ the pitch angle, and $I_{z z}$ the second moment of inertia of the wing section around the centre of gravity. The dynamics is given by

$$
I_{z z} \ddot{\theta}_{z}=M_{z}
$$

where $M_{z}$ is the resulting aerodynamic moment around the centre of gravity. Equation (3) can provide convincing insights into the application of the aerodynamic stability derivatives approach. Whereas a large body of work has been done on prescribed flight mechanics trajectories, ${ }^{6-8,28}$ a consistent study to assess the stability derivatives approach for a flying vehicle is missing, apart from a recent work by McCracken et al. ${ }^{29}$ This research work aims to expand previous investigations.

The aerodynamic moment around the centre of gravity can be expressed as

$$
M_{z}=M_{z}^{q . c .}+d\left(\cos \theta_{z} L-\sin \theta_{z} D\right)
$$

where $M_{z}^{q . c .}$ is the moment around the quarter chord, and $L$ and $D$ are the lift and drag forces, respectively. The term $d$ indicates the distance from the centre of gravity to the quarter chord point, positive if the latter is downstream of the centre of gravity. For the test case studied, $d=0.20 \mathrm{~m}$. A well-established approach for flight simulation is to consider the aerodynamic loads as a linear superposition of the effects due to flight conditions (angles of attack and sideslip, and Mach number), rotation of control surfaces, and motion rates (angular velocities around the three body axes). Details on the formulation can be found, for example, in Ref. ${ }^{8}$

As mentioned in the formulation section, the stability derivatives can be computed from a number of sources. For this case, the derivatives are obtained from a forced pitching motion CFD simulation, which was run at Mach number $0.14\left(U_{\infty}=50.0 \mathrm{~m} / \mathrm{s}\right.$ at sea level conditions), $\alpha_{0}=0.0 \mathrm{deg}, \alpha_{A}=1.0 \mathrm{deg}$, and $k=0.125$. The free-response simulations were run using both the CFD and UVLM methods. The three sources of stability derivative data are compared in Fig. 7.

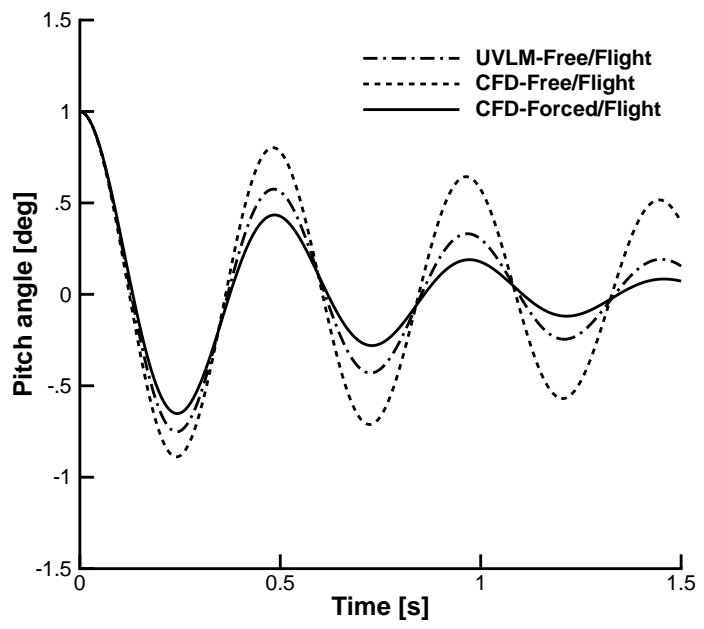

Figure 7. Time-domain response of a free-to-pitch two-dimensional wing section; comparison of sources of stability derivative terms; "Free" and "Forced" denote stability derivatives calculated from forced motions and free responses, respectively $\left(\alpha_{\infty}=1.0 \mathrm{deg}, U_{\infty}=50.0 \mathrm{~m} / \mathrm{s}, h=0.0 \mathrm{~m}\right.$, and $\left.\mathbf{R e}=3.5 \cdot 10^{6}\right)$

The static data used in these comparisons has been obtained from an aerodynamic tabular model as described in Ref. ${ }^{29}$ It is seen that there is a substantial difference between the three sources. The traditional approach of using forced motions predicts the greatest aerodynamic damping, whereas the CFD free response identification is the least well damped. The tabular model replay with the aerodynamic stability derivatives can be compared with the CFD and UVLM free responses. This is shown in Fig. 8. The error bars on this plot are applied to the tabular model prediction. They correspond to the range in pitch angle resulting from the source of the pitch damping derivative. It is seen that there is good agreement between the linear 
aerodynamic methods, e.g. UVLM and tabular models. There is, however, a noticeable difference from the unsteady CFD prediction. The phase shift seen is a result of unsteady history effects. Although for a modal problem, the influence of aerodynamic models on the dynamic response of a free-to-pitch wing has large impact.

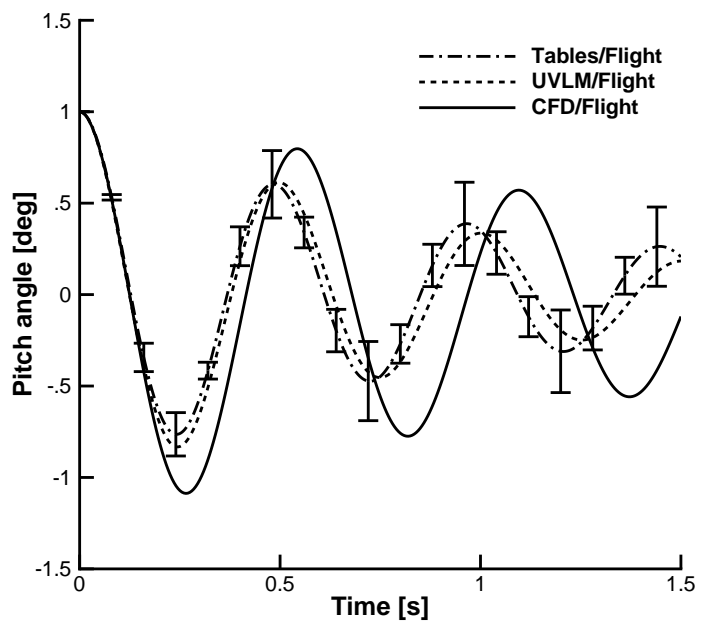

Figure 8. Time-domain response of a free-to-pitch two-dimensional wing section; comparison of modelling approaches; "Tables" denotes stability derivative approach $\left(\alpha_{\infty}=1.0 \mathrm{deg}, U_{\infty}=50.0 \mathrm{~m} / \mathrm{s}, h=0.0 \mathbf{m}\right.$, and Re $\left.=3.5 \cdot 10^{6}\right)$

\section{IV.C. Free-flying Test Case}

The test case considered here is for a free-flying wing. The model configuration and geometry are the same already presented. The wing has two rigid body degrees of freedom, one for the pitch rotation around the elastic axis and one for the motion in the vertical direction. Because linear aerodynamic models lack the ability to realistically represent drag contributions, it was decided to constrain the horizontal component of the motion to zero by introducing appropriate reaction forces. This allows to compare directly the responses computed by linear aerodynamic models with the CFD solution.

The time-domain responses of the pitch angle and vertical displacement for the same initial condition already discussed above are shown in Fig. 9. Numerical models predict a steady-state response well within a time window of $1.5 \mathrm{~s}$. However, simulations were run for a total of $5 \mathrm{~s}$ and the steady-state solutions showed no differences. The qualitative behaviour predicted by the three coupled models is similar. The time required for the pitch angle to reach its minimum value (at about $0.25 \mathrm{~s}$ ) is in good agreement between the UVLM and CFD solutions. As already observed in Fig. 6 and here confirmed, the wing response is the most damped in the case of thin aerofoil aerodynamics and the least damped when using CFD as source of the aerodynamic predictions. The quantitative differences are representative of considerably different levels of model complexities, realism, and costs.

Note that the steady-state solution is not unique and depends highly on the aerodynamic model used. With no gravity acting on the system, the free-flying wing reaches a steady-state equilibrium when the effective angle of attack is zero. For a two degrees of freedom system, the effective angle of attack is expressed as

$$
\alpha_{e f f}=\theta_{z}-\frac{\dot{y}}{U_{\infty}}
$$

where $\dot{y}$ is the velocity component in the vertical direction. From the above equation, it is apparent that the non-uniqueness of the steady-state solution comes down to an over-determined problem, with the values of rigid body pitch angle and vertical velocity component needed to cancel the combined effect, e.g. $\alpha_{e f f}=0$. 


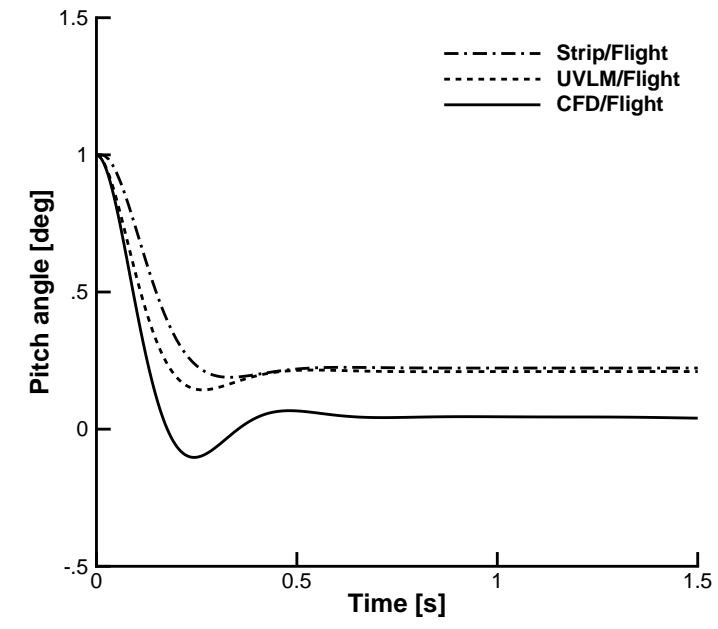

(a) Pitch angle

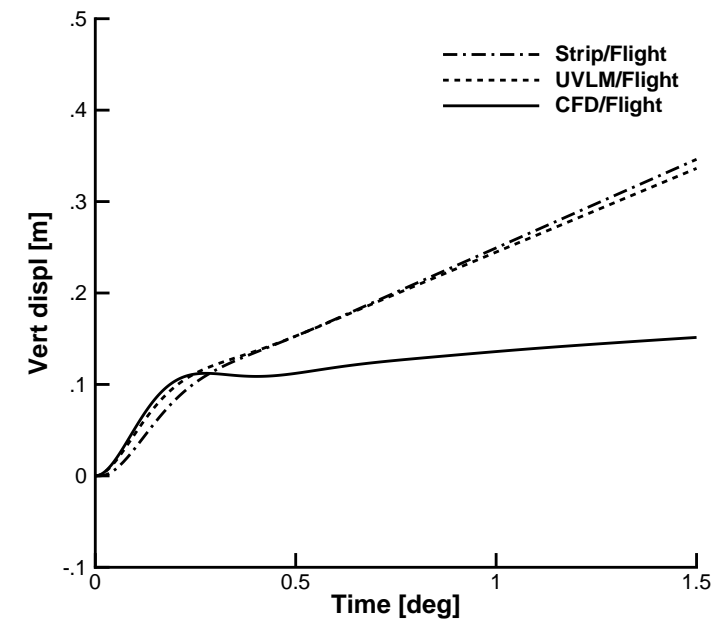

(b) Vertical displacement

Figure 9. Time-domain response of a free-flying two-dimensional wing section; "Strip" denotes two-dimensional thin aerofoil theory $\left(\alpha_{\infty}=1.0 \mathrm{deg}, U_{\infty}=50.0 \mathrm{~m} / \mathbf{s}, h=0.0 \mathbf{m}\right.$, and $\left.\mathbf{R e}=3.5 \cdot 10^{6}\right)$

\section{IV.C.1. Model based on Aerodynamic Stability Derivatives}

As for the single degree of freedom case, the free-flying wing can be run using the aerodynamic tabular model with stability derivatives. The same three sources of derivative have been used, this time calculating the lift derivatives as well as the pitching moment derivatives. A comparison of the three sources is shown in Fig. 10 for the pitch angle and vertical displacement.

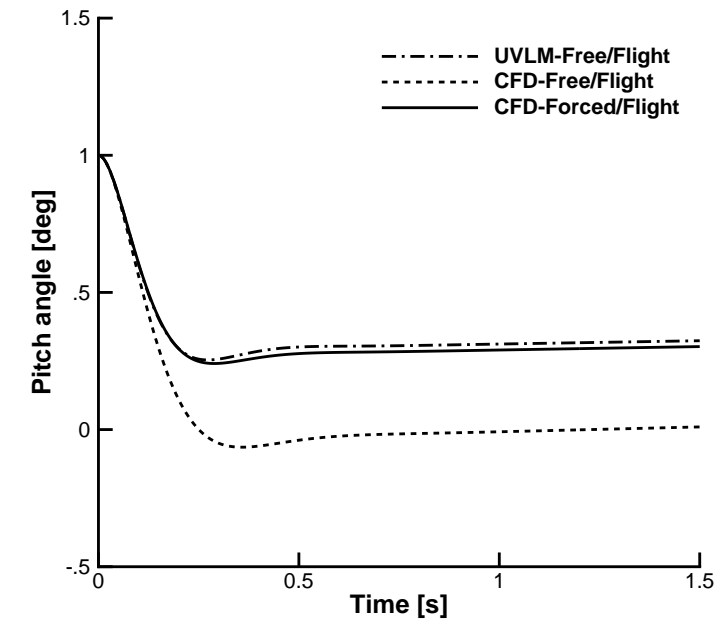

(a) Pitch angle

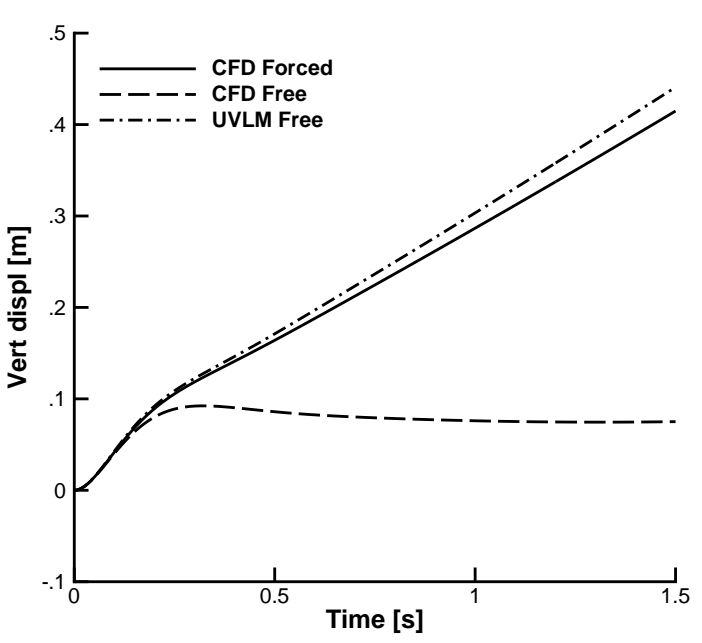

(b) Vertical displacement

Figure 10. Time-domain response of a free-flying two-dimensional wing section; comparison of sources of stability derivative terms; "Free" and "Forced" denote stability derivatives calculated from forced motions and free responses, respectively $\left(\alpha_{\infty}=1.0 \mathrm{deg}, U_{\infty}=50.0 \mathrm{~m} / \mathrm{s}, h=0.0 \mathrm{~m}\right.$, and Re $\left.=3.5 \cdot 10^{6}\right)$

The source of the derivative data again has a large effect on the simulated response. This is particularly noticeable in the vertical displacement plot. The linear methods have similar values, although the nonlinear free-response CFD is far from the other two. A comparison of the tabular response against the CFD and UVLM methods for the free-flying wing can be carried out. The results are shown in Fig. 11.

The error bars applied to the tabular simulation show the range of values as a result of the source of 


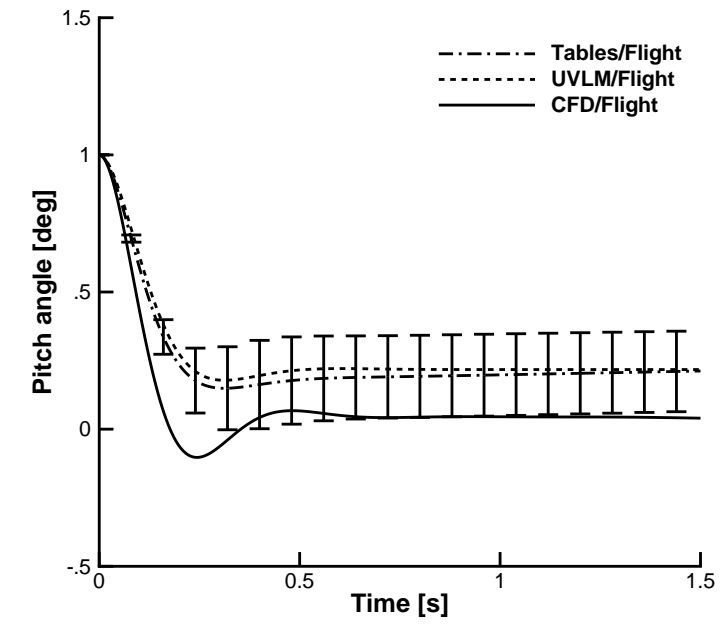

(a) Pitch angle

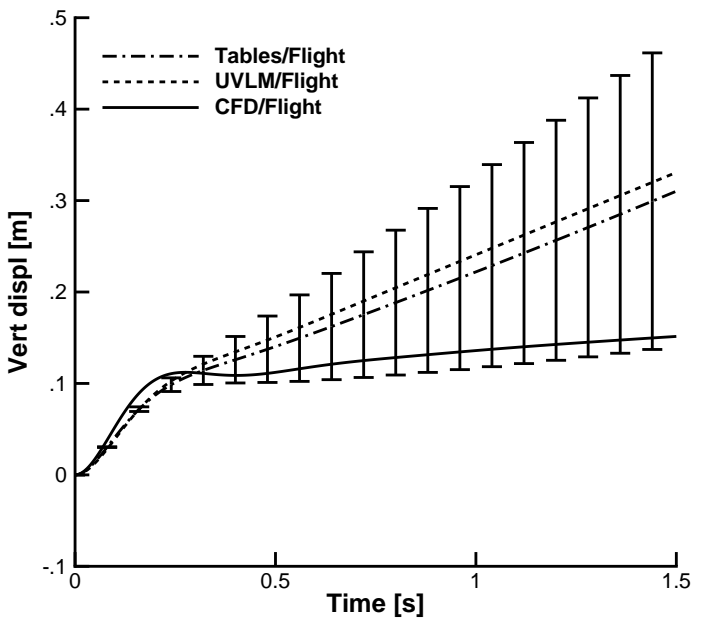

(b) Vertical displacement

Figure 11. Time-domain response of a free-flying two-dimensional wing section; comparison of modelling approaches; "Tables" denotes stability derivative approach $\left(\alpha_{\infty}=1.0 \mathrm{deg}, U_{\infty}=50.0 \mathrm{~m} / \mathrm{s}, h=0.0 \mathrm{~m}\right.$, and $\left.\mathbf{R e}=3.5 \cdot 10^{6}\right)$

stability derivative data. As with the free-to-pitch case, the linear methods are in good agreement with each other. However, the nonlinear CFD shows significant differences in both the pitch angle and vertical displacement. This is again due to the presence of history effects only being captured in the CFD model. In having the error bars, it is possible to see the uncertainty introduced in the simulations as a result of the aerodynamic model.

In both the free-to-pitch and free-flying cases, due to the free-response nature, errors at each step in the manoeuvre begin to accumulate. This is particularly noticeable for the vertical displacement. The errors seen in the simulations in this work are only over a period of $1.5 \mathrm{~s}$. These models are used in flight simulators and onboard control systems. These use values over time ranges of greater than just 1.5 seconds. This causes significant practical problems when it comes to selecting an aerodynamic model.

\section{Conclusions}

The work detailed in this paper investigates the impact of aerodynamic models on the dynamic response of a set of free-flying manoeuvres. The test case is an infinite-span aircraft wing. Future work will focus first on a modified configuration of the Goland wing, and then on a unmanned aerial vehicle geometry. Four aerodynamic models are used to assess the impact of model accuracies and costs on the predicted responses: two-dimensional thin aerofoil aerodynamics, unsteady vortex-lattice method, computational fluid dynamics, and the aerodynamic stability derivatives approach.

The paper expands previous works by the authors on assessing the limitations of aerodynamic tabular models for forced motions and replaying those motions in a time-accurate fashion using computational fluid dynamics. The objectives of this work are two-fold. First, a numerical framework that is currently developed for multi-disciplinary fluid/structure/flight analysis is validated for cases in which structural effects can be neglected. Two test cases are used for validation, and comparison with various aerodynamic sources is presented in all cases. With a well validated fluid/flight solver based on computational fluid dynamics as a background, the second objective is evaluating the differences in the dynamic response of a free-flying aircraft wing using a hierarchy of aerodynamic models. These differences are attributed to the aerodynamic models used, and their ability to realistically represent unsteady and time history effects. The well-established approach based on stability derivatives is found, in general, in good agreement with linear aerodynamic models, including cases in which the derivative values are estimated from computational fluid dynamics solutions. The uncertainty in the predicted trajectories of the free-flying wing for various aerodynamic models is large, and suggests careful considerations when performing control law synthesis. 


\section{Acknowledgements}

NDT, KJB, HH, and RP wish to acknowledge the support of the U.K. Engineering and Physical Sciences Research Council (EPSRC) grant EP/I014594/1 on Nonlinear Flexibility Effects on Flight Dynamics and Control of Next-Generation Aircraft.

\section{References}

${ }^{1}$ Kimberlin, R. D., Flight Testing of Fixed-Wing Aircraft, AIAA Educational Series, Reston, Virginia, USA, 2003.

${ }^{2}$ Barlow, J. B., William, H. R., and Pope, A., Low-Speed Wind Tunnel Testing, Third Edition, John Wiley \& Sons, Inc., 1999.

${ }^{3}$ Williams, J. E. and Vukelich, S. R., "The USAF Stability and Control Digital DATCOM," Tech. Rep. AFFDL-TR-793032, McDonnell Douglas Astronautics Company - St Louis Division, St. Louis, MO, 1979.

${ }^{4}$ Ghoreyshi, M., Badcock, K. J., Da Ronch, A., Vallespin, D., and Rizzi, A., "Automated CFD Analysis for the Investigation of Flight Handling Qualities," Mathematical Modelling of Natural Phenomena, Vol. 6, No. 3, 2011, pp. 166-188, doi: $10.1051 / \mathrm{mmnp} / 20116307$.

${ }^{5}$ Bryan, G. H., Stability in Aviation, MacMillan, London, 1911.

${ }^{6}$ Da Ronch, A., Vallespin, D., Ghoreyshi, M., and Badcock, K. J., "Evaluation of Dynamic Derivatives Using Computational Fluid Dynamics," AIAA Journal, Vol. 50, No. 2, 2012, pp. 470-484, doi: 10.2514/1.J051304.

${ }^{7}$ Da Ronch, A., McCracken, A. J., Badcock, K. J., Widhalm, M., and Campobasso, M. S., "Linear Frequency Domain and Harmonic Balance Predictions of Dynamic Derivatives," Journal of Aircraft, Vol. 50, No. 3, 2013, pp. 694-707, doi: 10.2514/1.C031674.

${ }^{8}$ Da Ronch, A., Ghoreyshi, M., and Badcock, K. J., "On the Generation of Flight Dynamics Aerodynamic Tables by Computational Fluid Dynamics," Progress in Aerospace Sciences, Vol. 47, No. 8, 2011, pp. 597-620, doi: $10.1016 /$ j.paerosci.2011.09.001.

${ }^{9}$ Ghoreyshi, M., Cummings, R. M., Da Ronch, A., and Badcock, K. J., "Transonic Aerodynamic Loads Modeling of X-31 Aircraft Pitching Motions," AIAA Journal, 2013, doi: 10.2514/1.J052309.

${ }^{10}$ Da Ronch, A., Badcock, K. J., Wang, Y., Wynn, A., and Palacios, R. N., "Nonlinear Model Reduction for Flexible Aircraft Control Design," AIAA Atmospheric Flight Mechanics Conference, AIAA Paper 2012-4404, Minneapolis, MN, 13-16 August 2012, doi: 10.2514/6.2012-4404.

${ }^{11}$ Murua, J., Palacios, R., and Graham, J. M. R., "Assessment of Wake-Tail Interference Effects on the Dynamics of Flexible Aircraft," AIAA Journal, Vol. 50, No. 7, 2012, pp. 1575-1585, doi: 10.2514/1.J051543.

${ }^{12}$ Murua, J., Palacios, R., and Graham, J. M. R., "Applications of the unsteady vortex-lattice method in aircraft aeroelasticity and flight dynamics," Progress in Aerospace Sciences, Vol. 55, 2012, pp. 46-72, doi: 10.1016/j.paerosci.2012.06.001.

${ }^{13}$ Palacios, R., Murua, J., and Cook, R., "Structural and Aerodynamic Models in the Nonlinear Flight Dynamics of Very Flexible Aircraft," AIAA Journal, Vol. 48, No. 11, 2010, pp. 2648-2559, doi: 10.2514/1.52446.

${ }^{14}$ Katz, J. and Plotkin, A., Low-Speed Aerodynamics, Cambridge Aerospace Series, Cambridge University Press, New York, NY, USA, 2nd ed., 2001.

${ }^{15}$ Kennett, D. J., Timme, S., Angulo, J., and Badcock, K. J., "An Implicit Meshless Method for Application in Computational Fluid Dynamics," International Journal for Numerical Methods in Fluids, Vol. 71, No. 8, 2013, pp. 1007-1028, doi: $10.1002 /$ fld.3698.

${ }^{16}$ Kennett, D. J., Timme, S., Angulo, J., and Badcock, K. J., "Semi-Meshless Stencil Selection for Anisotropic Point Distributions," International Journal of Computational Fluid Dynamics, Vol. 26, No. 9-10, 2012, pp. 463-487, doi: 10.1080/10618562.2012.744450.

${ }^{17}$ Da Ronch, A., Tantaroudas, N. D., Timme, S., and Badcock, K. J., "Model Reduction for Linear and Nonlinear Gust Loads Analysis," 54th AIAA/ASME/ASCE/AHS/ASC Structures, Structural Dynamics, and Materials Conference, AIAA Paper 2013-1492, Boston, MA, 08-11 April 2013, doi: 10.2514/6.2013-1492.

${ }^{18}$ Hesse, H. and Palacios, R., "Consistent structural linearisation in flexible-body dynamics with large rigid-body motion," Computers \& Structures, Vol. 110-111, 2012, pp. 1-14, doi: 10.1016/j.compstruc.2012.05.011.

${ }^{19}$ Géradin, M. and Cardona, A., Flexible Multibody Dynamics: A Finite Element Approach, John Wiley \& Sons Ltd, Chichester, UK, 2001.

${ }^{20}$ Géradin, M. and Rixen, D., Mechanical vibrations: theory and application to structural dynamics, John Wiley, Chichester, UK, 2nd ed., 1997.

${ }^{21}$ Peterson, P., "F2PY: a Tool for Connecting Fortran and Python Programs," International Journal of Computational Science and Engineering, Vol. 4, No. 4, 2009, pp. 296-305, doi: 10.1504/ijcse.2009.029165.

${ }^{22}$ Dalcín, L., Paz, R., Storti, M., and D'Elía, J., "MPI for Python: Performance Improvements and MPI-2 Extensions," Journal of Parallel and Distributed Computing, Vol. 68, No. 5, 2008, pp. 655-662, doi: 10.1016/j.jpdc.2007.09.005.

${ }^{23}$ Quaranta, G., Masarati, P., and Mantegazza, P., "A Conservative Mesh-Free Approach for Fluid-Structure Interface Problems," International Conference for Coupled Problems in Science and Engineering, Greece, 2005.

${ }^{24}$ Yates, E. C., "AGARD Standard Aeroelastic Configurations for Dynamic Response. I - Wing 445.6," Tech. Rep. NASA$\mathrm{R}-765,1988$.

${ }^{25} \mathrm{Da}$ Ronch, A., Tantaroudas, N. D., and Badcock, K. J., "Reduction of Nonlinear Models for Control Applications," 54th AIAA/ASME/ASCE/AHS/ASC Structures, Structural Dynamics, and Materials Conference, AIAA Paper 2013-1491, Boston, MA, 08-11 April 2013, doi: 10.2514/6.2013-1491. 
${ }^{26}$ Witteveen, J. A. S. and Bijl, H., "Explicit Mesh Deformation using Inverse Distance Weighting Interpolation," 19th AIAA Computational Fluid Dynamics Conference, AIAA Paper 2009-3996, San Antonio, TX, 2009, doi: 10.2514/6.2009-3996.

${ }^{27}$ McCroskey, W. J. and Pucci, S. L., "Viscous-Inviscid Interaction on Oscillating Airfoils in Subsonic Flows," 19 th AIAA Aerospace Sciences Meeting, AIAA Paper 1981-51, St. Louis, MO, 12-15 January 1981, doi: 10.2514/6.1981-51.

${ }^{28}$ Vallespin, D., Badcock, K. J., Da Ronch, A., White, M., Perfect, P., and Ghoreyshi, M., "Computational Fluid Dynamics Framework for Aerodynamic Model Assessment," Progress in Aerospace Sciences, Vol. 52, No. 0, 2012, pp. 2-18, doi: $10.1016 /$ j.paerosci.2011.12.004.

${ }^{29}$ McCracken, A. J., Kennett, D. J., Badcock, K. J., and Da Ronch, A., "Assessment of Tabular Models using CFD," AIAA Atmospheric Flight Mechanics Conference, AIAA Paper 2013-4978, Boston, MA, 19-22 August 2013, doi: 10.2514/6.2013-4978. 